\title{
Nrdp1-mediated degradation of BRUCE decreases cell viability and induces apoptosis in human 786-O renal cell carcinoma cells
}

\author{
SHAO-JUN CHEN* ${ }^{*}$ JIAN-HAI LIN* ${ }^{*}$ XU-DONG YAO, BO PENG, YUN-FEI XU, MIN LIU and JUN-HUA ZHENG \\ Department of Urology, Shanghai Tenth People's Hospital, Tongji University, Shanghai 200072, P.R. China
}

Received February 5, 2015; Accepted March 30, 2016

DOI: $10.3892 /$ etm.2016.3356

\begin{abstract}
Neuregulin receptor degradation protein-1 (Nrdp1) is involved in a plethora of cellular processes and plays an essential role in the development and progression of human cancers. However, its role in renal cell carcinoma (RCC) remains unclear. Therefore, the present study aimed to explore the biological significance of Nrdp1 in RCC. Western blot analyses of tissue samples from 24 patients with primary RCC revealed lower Nrdp1 and higher baculovirus inhibitor of apoptosis repeat-containing ubiquitin-conjugating enzyme (BRUCE) protein levels in RCC tissues compared with adjacent normal tissues. In addition, MTT and apoptosis assays demonstrated that Nrdp1 overexpression resulted in decreased cell viability and enhanced apoptosis in RCC 786-O cells; conversely, Nrdp1 knockdown increased 786-O cell viability and inhibited apoptosis. Further analysis showed that BRUCE downregulation partially attenuated the effects of Nrdp1 knockdown on RCC cell viability and apoptosis. Moreover, an inverse association was obtained between BRUCE and Nrdp1 protein levels. These findings suggest that Nrdp1-mediated degradation of BRUCE decreases cell viability and induces apoptosis in RCC cells, highlighting Nrdp1 as a potential target for RCC treatment.
\end{abstract}

\section{Introduction}

Renal cell carcinoma (RCC) is the most common type of kidney cancer with an incidence of 5-10 per 100,000; it accounts for $\sim 3 \%$ of all cancer diagnoses in adults $(1,2)$. In the United States alone, $>65,000$ individuals were diagnosed with RCC in 2013, with $>13,600$ mortalities recorded (3). At

Correspondence to: Dr Min Liu or Dr Jun-Hua Zheng, Department of Urology, Shanghai Tenth People's Hospital, Tongji University, 301 Yanchang Road, Shanghai 200072, P.R. China

E-mail: kidneyliu@163.com

E-mail: zhengjh0471@sina.com

*Contributed equally

Key words: Nrdp1, BRUCE, renal cell carcinoma, viability, apoptosis present, surgical resection remains the most common treatment method for renal cancer because of its poor response to chemotherapy and radiotherapy; however, nearly $30 \%$ of patients develop metastatic disease following surgery, showing a median survival time of only 13 months $(4,5)$. Furthermore, the 5-year survival rate of renal cancer is estimated to be $\sim 55 \%$, and that of metastatic renal cell carcinoma is $\sim 10 \%$ (6). Despite significant advances in the diagnosis and treatment of RCC, its mechanism of carcinogenesis is largely unknown and the prognosis remains poor. Further understanding of RCC pathogenesis and the identification of novel molecules involved in its progression may provide new strategies for treatment.

The enzyme neuregulin receptor degradation protein-1 (Nrdp1), a RING finger domain-containing E3 ubiquitin ligase, is a member of a family of E3 ubiquitin ligases designated tripartite motif/RING finger, B-box, and coiled-coil proteins, which are involved in numerous cellular processes such as apoptosis, cell cycle regulation and viral response $(7,8)$. Nrdp1 was originally found to be involved in the ubiquitination and proteasomal degradation of Erb-B2 receptor tyrosine kinase 3 (ErbB3), a member of the epidermal growth factor receptor (EGFR) family (9). In the past decades, Nrdp1-mediated degradation of ErbB3 has been shown to be correlated with a number of diseases, such as human breast and colorectal cancers $(10,11)$. Furthermore, Nrdp1 is also implicated in the ubiquitination and degradation of two other E3 ubiquitin ligases, namely baculovirus inhibitor of apoptosis repeat (BIR)-containing ubiquitin-conjugating enzyme (BRUCE) (an apoptosis inhibitor) and parkin, which is involved in the pathogenesis of Parkinson's disease $(12,13)$. Nrdp1 has been reported to increase Toll-like receptor-mediated interferon- $\beta$ production by suppressing myeloid differentiation primary response gene 88 and activating TANK-binding kinase 1 (14). However, the role of Nrdp1 in RCC development and progression remains largely unknown.

BRUCE, also known as apollon, is a giant (528 kDa) membrane-associated protein that contains a BIR domain at its N-terminal region. It is a member of the inhibitor of apoptosis (IAP) family of proteins, and inhibits caspase activity and apoptosis via its BIR domain $(15,16)$. Qiu and Goldberg demonstrated that decreasing BRUCE levels by proteasomal degradation or RNA interference promotes cell apoptosis (17). In addition, Hao et al indicated that BRUCE is essential in preventing second mitochondria-derived activator of caspase 
(SMAC)-induced apoptosis, promoting ubiquitination and proteasomal degradation of SMAC (18). Consistent with this, Chen et al (15) reported that BRUCE is upregulated in certain brain cancers (gliomas), suggesting that this protein plays a role in tumorigenesis of gliomas by protecting cells from apoptosis. Previous studies have revealed that Nrdp1 overexpression results in increased cell apoptosis through the induction of BRUCE ubiquitination and proteasomal degradation; consistent with this, decreasing Nrdp1 levels by RNA interference causes decreased cell apoptosis, suggesting that Nrdp1-mediated degradation of BRUCE represents a novel mechanism of apoptosis $(12,19)$.

The present study aimed to investigate the roles of Nrdp1 and one of its targets, BRUCE, in RCC. First, the protein expression levels of Nrdpl and BRUCE were assessed in RCC and adjacent normal tissues by western blot analysis. Secondly, Nrdp1 was overexpressed or silenced in 786-O RCC cells to determine the biological role of Nrdp1. Finally, BRUCE-specific small interfering RNA (si-BRUCE) was transfected into cells to assess whether the levels of BRUCE are associated with the effects of Nrdp1 observed in RCC.

\section{Materials and methods}

Tissue specimens. A total of 24 paired tumor specimens and adjacent normal tissues were obtained from patients with primary RCC who had undergone radical nephrectomy at the Department of Urology, Shanghai Tenth People's Hospital, Tongji University (Shanghai, China), between 2009 and 2013. None of the patients received preoperative treatment. Following surgical resection, samples were placed immediately into liquid nitrogen for cryopreservation until use; tumor specimens were all confirmed by postoperative pathological analysis. This study was approved by the Ethics Committee of the Shanghai Tenth People's Hospital, and written informed consent was obtained from all patients.

Cell culture and transfection. The human RCC cell line 786-O was purchased from American Type Culture Collection (ATCC; Manassas, VA, USA). Cells were cultured at $37^{\circ} \mathrm{C}$ in a humid environment containing $5 \% \mathrm{CO}_{2}$ in Dulbecco's modified Eagle's medium (Invitrogen; Thermo Fisher Scientific, Inc., Waltham, MA, USA) supplemented with $10 \%$ fetal bovine serum (Thermo Fisher Scientific, Inc.), $50 \mathrm{U} / \mathrm{ml}$ penicillin and $50 \mu \mathrm{g} / \mathrm{ml}$ streptomycin.

Transfection was performed using the Lipofectamine 2000 transfection reagent (Invitrogen; Thermo Fisher Scientific, Inc.) following the manufacturer's protocol. An Nrdp1 construct was generated by subcloning the human Nrdp1 cDNA into the expression vector pcDNA3.1-EGFR (both Shanghai GenePharma, Co., Ltd., Shanghai, China). For Nrdp1 silencing, the corresponding human small interference short hairpin RNA (shRNA; Shanghai GenePharma, Co., Ltd.) sequences were cloned into pcDNA3.1-EGFR to generate a shRNA construct (shNrdp1). Cells transfected with null plasmid were used as controls. For functional analysis of BRUCE, shNrdp1 and si-BRUCE (Shanghai GenePharma, Co., Ltd.) were simultaneously transfected into pretreated cells. The following sequence was used for si-BRUCE: 5'-CCU GACAAUGCAGAAGGAAUCCAU-3'.
RNA isolation and reverse transcription-quantitative polymerase chain reaction ( $R T-q P C R)$. Total RNA was extracted from pretreated cells using TRIzol reagent (Invitrogen; Thermo Fisher Scientific, Inc.) according to the manufacturer's protocol. The RNA samples were reverse-transcribed into cDNA using the PrimeScript ${ }^{\mathrm{TM}}$ RT-PCR kit (Takara Biotechnology Co., Ltd., Dalian, China). The relative gene expression levels of Nrdp1 or BRUCE were evaluated by RT-qPCR using an Applied Biosystems 7900HT Fast Real-Time PCR System (Thermo Fisher Scientific, Inc.) with $1.0 \mu \mathrm{l}$ cDNA and SYBR Green Real-time PCR Master mix (Takara Biotechnology Co., Ltd.). The PCR amplification was carried out for 40 cycles of $94^{\circ} \mathrm{C}$ for $30 \mathrm{sec}, 60^{\circ} \mathrm{C}$ for $30 \mathrm{sec}$, and $72^{\circ} \mathrm{C}$ for $30 \mathrm{sec}$. The following primers were used: Nrdp1, forward 5'-TGAACC GACGCTACTATGAGAACT-3' and reverse 5'-CTGGTT CTCACAGGCCATCAC-3'; BRUCE, forward 5'-CTCAGT CAGTCCTGCCTCATC-3' and reverse 5'-CTCAGCAGTCCT CTGGATGTC-3'; glyceraldehyde 3-phosphate dehydrogenase (GAPDH), forward 5'-GTAAGACCCCTGGACCACCA-3' and reverse 5'-CAAGGGGTCTACATGGCAACT-3'. The relative expression levels were normalized to GAPDH and analyzed using the $2^{-\Delta \Delta C q}$ method (20). All experiments were carried out in triplicate.

3-(4,5-dimethylthiazol-2-yl)-2,5-diphenyl tetrazolium bromide (MTT) assay. Cell viability was measured by the MTT assay (Sigma-Aldrich, St. Louis, MO, USA) according to the manufacturer's protocol. Briefly, following effective transfection, 786-O cells were seeded into 96-well plates at a density of $5 \times 10^{3}$ cells per well. The plates were incubated at $37^{\circ} \mathrm{C}$ in a humidified atmosphere containing $5 \% \mathrm{CO}_{2}$ for 24 , 48, 72 and $96 \mathrm{~h}$, respectively. Afterwards, $20 \mu \mathrm{l}$ MTT solution ( $5 \mathrm{mg} / \mathrm{ml}$ ) was added to each well, followed by $4 \mathrm{~h}$ incubation at $37^{\circ} \mathrm{C}$. After careful removal of the medium, $150 \mu \mathrm{l}$ dimethyl sulfoxide (Sigma-Aldrich) was used to solubilize the crystals for 15 min. Finally, cell viability was assessed spectrophotometrically at $490 \mathrm{~nm}$. Each experiment was repeated at least three times.

Apoptosis assay. Cell apoptosis was assessed using a fluorescein isothiocyanate (FITC)-Annexin V Apoptosis Detection kit (BD Biosciences, San Jose, CA, USA) following the manufacturer's guidelines. Transfected cells were cultured in serum-free medium. After trypsinization, cells were washed twice with phosphate-buffered saline (TBS), and stained with FITC-Annexin V and propidium iodide (PI; BD Biosciences) in the dark for $15 \mathrm{~min}$ at room temperature. Finally, apoptotic cells were quantified by flow cytometry using a BD FACSCalibur flow cytometer (BD Biosciences). The experiments were repeated at least three times.

Western blot analysis. Total protein was extracted from tissues (RCC tumors and adjacent normal tissues) and cultured cells with radioimmunoprecipitation assay (RIPA) lysis buffer [50 mM Tris/HCl, pH 7.4, $150 \mathrm{mM} \mathrm{NaCl,} 1 \%$ NP-40, 0.1\% sodium dodecyl sulfate (SDS)] containing a protease inhibitor cocktail(Sigma-Aldrich) on ice.The supernatants were collected following centrifugation for $20 \mathrm{~min}$ at $25,155 \mathrm{x} \mathrm{g}$ and $4^{\circ} \mathrm{C}$. Protein lysates were subjected to $10 \%$ SDS-polyacrylamide gel electrophoresis and transferred onto polyvinylidene difluoride 
A

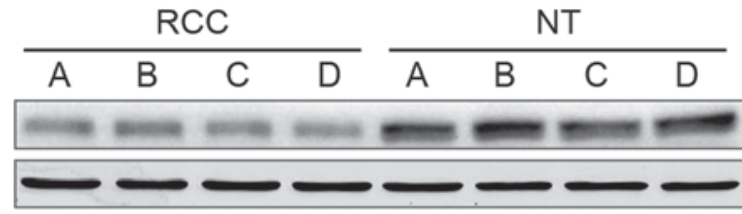

patient

Nrdp1

$\beta$-actin

C

\begin{tabular}{|c|c|c|c|c|c|c|c|c|}
\hline \multicolumn{4}{|c|}{ RCC } & \multicolumn{4}{|c|}{ NT } & \\
\hline a & b & c & d & a & $b$ & c & d & patient \\
\hline 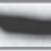 & 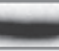 & $=$ & - & - & 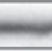 & $=$ & - & BRUCE \\
\hline
\end{tabular}

B

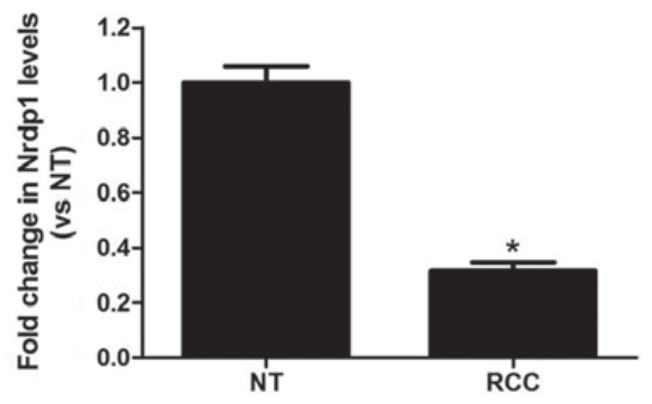

D

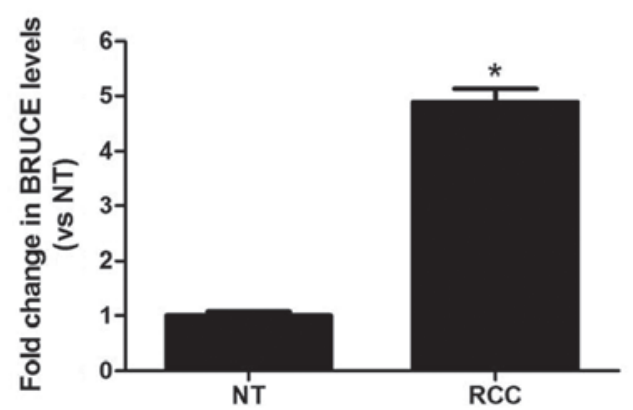

Figure 1. RCC specimens show decreased Nrdp1 and increased BRUCE protein levels. Western blot analysis was performed on paired RCC (RCC) and adjacent normal tissues (NT) from the same patients. (A and B) Nrdp1 levels were significantly decreased in RCC specimens compared with adjacent normal tissues, shown by (A) representative images and (B) quantification. (C and D) BRUCE levels were significantly increased in RCC samples compared with adjacent normal tissues, shown by (C) representative images and (D) quantification. Data are the mean \pm standard deviation from three independent experiments. ${ }^{*} \mathrm{P}<0.05$ vs. the NT group. RCC, renal cell carcinoma; Nrdp1, neuregulin receptor degradation protein-1; BRUCE, baculovirus inhibitor of apoptosis repeat-containing ubiquitin-conjugating enzyme.

membranes (Bio-Rad Laboratories, Inc., Hercules, CA, USA). For immunoblotting, the membranes were blocked with $5 \%$ non-fat milk in TBS and Tween 20 at room temperature for $1 \mathrm{~h}$ and incubated overnight at $4^{\circ} \mathrm{C}$ with mouse anti-Nrdp1 (1:500; cat. no. sc-374120; Santa Cruz Biotechnology, Inc., Dallas, TX, USA), mouse anti-BRUCE (1:1,000; cat. no. 611192; BD Biosciences) and mouse anti- $\beta$-actin $(1: 5,000$; cat. no. ab8226; Abcam, Cambridge, UK) monoclonal antibodies. After three washes, blots were incubated with horseradish peroxidase-conjugated goat anti-mouse secondary antibody (1:10,000; cat. no. ab97040; Abcam) for $2 \mathrm{~h}$ at room temperature. Finally, protein bands were detected using an enhanced chemiluminescence ECL kit (EMD Millipore, Billerica, MA, USA) and quantified using Image J 1.x software (National Institutes of Health, Bethesda, MD, USA). $\beta$-actin was used as an internal control, and three independent experiments were performed.

Statistical analysis. Data presented are the mean \pm standard deviation (SD) from three independent experiments. Statistical analyses were performed with GraphPad Prism software, version 5.0 (GraphPad Software, Inc., La Jolla, CA, USA). Groups were compared using a Student's t-test (two groups) or one-way analysis of variance (ANOVA; more than two groups). $\mathrm{P}<0.05$ was considered to indicate a statistically significant difference.

\section{Results}

Decreased Nrdpl and increased BRUCE levels in RCC. To determine whether Nrdp1 and BRUCE expression levels were altered in RCC specimens, western blot analysis was performed on paired RCC tumor and adjacent normal tissues from the same patients. As shown in Fig. 1, Nrdp1 levels were significantly lower in RCC tumors compared with adjacent normal tissues, while the levels of BRUCE were significantly higher $(\mathrm{P}<0.05)$. This suggests that both Nrdp1 and BRUCE may be involved in RCC progression.

Nrdp1 levels affect RCC cell viability and apoptosis. In order to investigate the biological role of Nrdp1 in RCC cells, in vitro assays were carried out using the human RCC 786-O cell line. Nrdp1 was overexpressed or silenced in 786-O cells. The overexpression of Nrdp1, which was confirmed by RT-qPCR and western blotting (Fig. 2A and B), resulted in significantly decreased cell viability in the MTT assay, and significantly increased apoptosis in 786-O cells as evaluated by flow cytometry ( $\mathrm{P}<0.05$; Fig. $2 \mathrm{C}$ and $\mathrm{D})$. Conversely, Nrdp1 knockdown using shNrdp1, also confirmed by RT-qPCR and western blotting (Fig. 3A and B), caused the cell viability to increase and reduced apoptosis in the 786-O cells (Fig. 3C and D). These results indicate that $\mathrm{Nrdp1}$ may act as a tumor suppressor in RCC.

Nrdpl affects RCC cell viability and apoptosis, likely by degrading BRUCE. Considerable evidence suggests that BRUCE is a target of Nrdp1, and that Nrdp1-mediated degradation of BRUCE is correlated with cell apoptosis in human cancers $(12,19)$. To verify these findings in RCC, the small interfering RNA si-BRUCE was transfected into 786-O cells with Nrdp1 knockdown. Reduced levels of BRUCE expression following transfection were confirmed by RT-qPCR and 
A

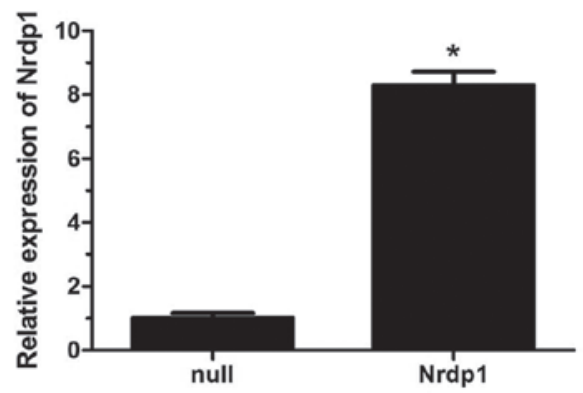

C

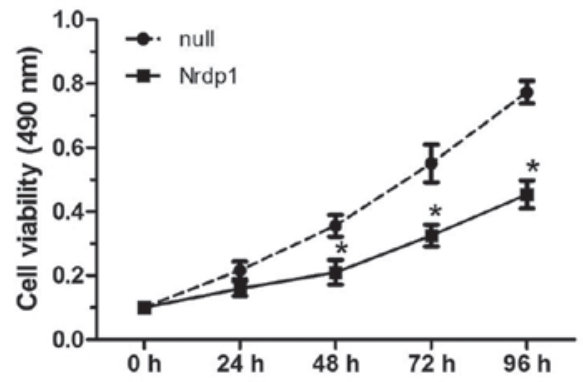

B

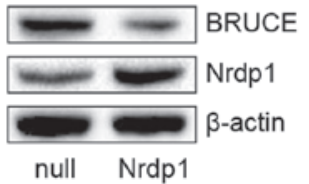

D

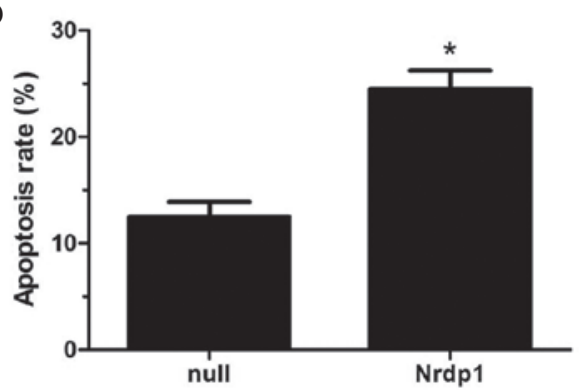

Figure 2. Nrdp1 overexpression decreases viability and induces apoptosis in 786-O cells. (A) Relative Nrdp1 RNA expression levels in 786-O cells with Nrdp1 overexpression, determined by reverse transcription-quantitative polymerase chain reaction. (B) Nrdp1 and BRUCE protein levels in 786-O cells overexpressing Nrdp1. (C) Overexpression of Nrdp1 significantly decreases 786-O cell viability. (D) Overexpression of Nrdp1 markedly induces apoptosis in 786-O cells. Data are the mean \pm standard deviation from three independent experiments. ${ }^{*} \mathrm{P}<0.05$ vs. the null group. Nrdp1, neuregulin receptor degradation protein-1; BRUCE, baculovirus inhibitor of apoptosis repeat-containing ubiquitin-conjugating enzyme.

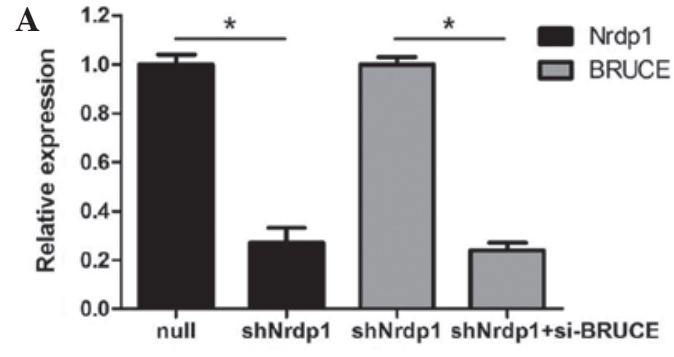

C

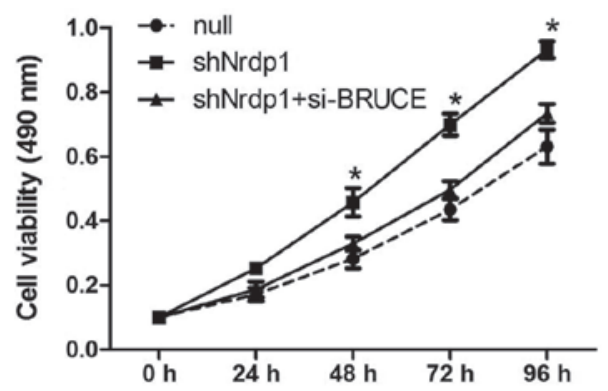

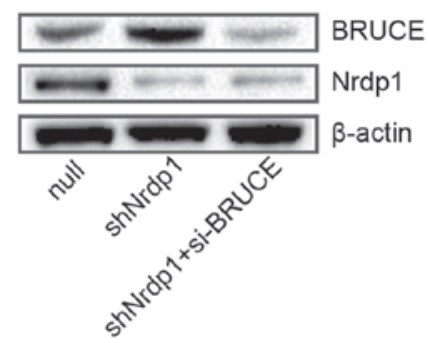

D

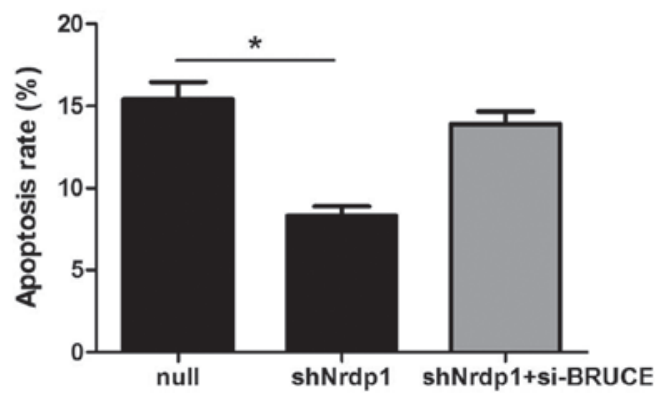

Figure 3. Knockdown of Nrdp1 increases viability and inhibits apoptosis in 786-O cells; the effects are partially attenuated by BRUCE downregulation. (A) Relative Nrdp1 or BRUCE RNA expression levels in 786-O cells, respectively, following Nrdp1 knockdown or BRUCE downregulation, as determined by reverse transcription-quantitative polymerase chain reaction ("P<0.05). (B) Nrdp1 and BRUCE protein levels in 786-O cells after Nrdp1 knockdown or BRUCE downregulation. (C) Nrdp1 knockdown increases cell viability in $786-\mathrm{O}$ cells (" $\mathrm{P}<0.05$ vs. the null group)., while the effect is partially attenuated by BRUCE downregulation. (D) Nrdp1 knockdown inhibits apoptosis in 786-O cells ( $\mathrm{P}<0.05)$, while the effect is partially attenuated by BRUCE downregulation. Data are mean \pm standard deviation from three independent experiments. Nrdp1, neuregulin receptor degradation protein-1; BRUCE, baculovirus inhibitor of apoptosis repeat-containing ubiquitin-conjugating enzyme; shNrdp1, short hairpin Nrdp1; si-BRUCE, BRUCE-specific small interfering RNA.

western blot analysis (Fig. 3A and B). Notably, the effects of Nrdp1 knockdown on the viability and apoptosis of 786-O cells were partially attenuated by BRUCE silencing (Fig. 3C and D). Moreover, the levels of BRUCE protein exhibited an inverse association with Nrdp1 protein levels as shown by western blot analysis (Figs. 2B and 3B). These findings suggest that Nrdp1 may affect the cell viability and apoptosis of RCC via the degradation of BRUCE. 


\section{Discussion}

$\mathrm{RCC}$ is the third most common urological cancer after prostate and bladder cancers, but has the highest mortality rate $(21,22)$. More seriously, its incidence and mortality rates are on the rise around the globe. As this cancer has a rather poor prognosis, the identification of novel molecules involved in RCC progression that may provide new strategies for therapeutic intervention would be valuable. Previous studies have shown that the Nrdp1-mediated degradation of BRUCE induces cell apoptosis, and is closely associated with the progression of human cancers $(12,19)$. It is acknowledged that multiple genetic or epigenetic regulations in RCC carcinogenesis and progression are correlated with cell viability and apoptosis $(23,24)$. Hence, Nrdp1-mediated degradation of BRUCE may also affect cell viability and apoptosis in RCC.

In the present study, the biological significance of Nrdp1 and one of its targets, BRUCE, in RCC were investigated. Significantly lower Nrdp1 expression levels were found in RCC specimens compared with the corresponding normal tissues obtained from patients. In addition, a significant upregulation of BRUCE was observed in RCC specimens. Functional analyses revealed that the forced overexpression of $\mathrm{Nrdp} 1$ markedly decreased cell viability and induced apoptosis in the human RCC 786-O cell line; conversely, Nrdp1 knockdown markedly increased cell viability and inhibited apoptosis in 786-O cells. These data suggest that Nrdp1 is downregulated in RCC and that the low Nrdp1 levels may be associated with RCC development and progression.

Apoptosis is an ordered and orchestrated cellular process that plays a critical role in normal tissue development and homeostasis. Nevertheless, apoptosis inhibition is considered to contribute to carcinogenesis and progression of human cancers $(25,26)$. BRUCE functions as an inhibitor of apoptosis, and is regulated by Nrdp1-mediated degradation $(12,27)$. Therefore, we hypothesized that BRUCE may be associated with the effects of Nrdp1 on RCC cell viability and apoptosis. In vitro assays showed that the downregulation of BRUCE partially attenuates the effects of Nrdp1 knockdown on RCC cell viability and apoptosis. Moreover, the levels of BRUCE exhibited an inverse association with Nrdpl levels as demonstrated by western blot analysis. These findings suggest that Nrdp1 may affect RCC cell viability and apoptosis through the proteasomal degradation of BRUCE.

As a limitation of the present study, the downstream mechanism through which BRUCE inhibits cell apoptosis in RCC was not explored. It has previously been reported that BRUCE binds to, ubiquitinates and facilitates the proteasomal degradation of SMAC and caspase-9, which play key roles in the initiation of apoptosis, thereby inhibiting cell apoptosis in mammals (18). Furthermore, the tumor suppressor p53 has been identified as a downstream effector of BRUCE, and the induction of apoptosis following BRUCE knockdown has been shown to be associated with upregulation and nuclear localization of p53 (28). Therefore, further study is warranted to investigate how the Nrdp1-mediated degradation of BRUCE modulates cell viability and apoptosis in RCC.

The present study demonstrates for the first time, to the best of our knowledge, that Nrdp1 is significantly decreased in RCC specimens compared with adjacent normal tissues, and decreases viability while inducing apoptosis in RCC cells. In addition, the biological significance of Nrdp1 in RCC cells is associated with its proteasomal degradation of BRUCE. These findings suggest that the Nrdp1-mediated degradation of BRUCE decreases cell viability and induces apoptosis in RCC cells, highlighting Nrdp1 as a potential target for RCC treatment.

\section{Acknowledgements}

This study was partially supported by grants from the National Natural Science Foundation of China (no. 81000311 and no. 81270831$)$.

\section{References}

1. Yang J, Yang J, Gao Y, Zhao L, Liu L, Qin Y, Wang X, Song T and Huang C: Identification of potential serum proteomic biomarkers for clear cell renal cell carcinoma. PLoS One 9: e111364, 2014.

2. Siegel R, Naishadham D and Jemal A: Cancer statistics, 2013. CA Cancer J Clin 63: 11-30, 2013.

3. Lasseigne BN, Burwell TC, Patil MA, Absher DM, Brooks JD and Myers RM: DNA methylation profiling reveals novel diagnostic biomarkers in renal cell carcinoma. BMC Med 12: 235, 2014.

4. Janowitz T, Welsh SJ, Zaki K, Mulders P and Eisen T: Adjuvant therapy in renal cell carcinoma - past, present, and future. Semin Oncol 40: 482-491, 2013.

5. Li W, Liu M, Xu YF, Feng Y, Che JP, Wang GC and Zheng JH: Combination of quercetin and hyperoside has anticancer effects on renal cancer cells through inhibition of oncogenic microRNA-27a. Oncol Rep 31: 117-124, 2014.

6. Zhang HM, Yang FQ, Yan Y, Che JP and Zheng JH: High expression of long non-coding RNA SPRY4-IT1 predicts poor prognosis of clear cell renal cell carcinoma. Int J Clin Exp Pathol 7: 5801-5809, 2014

7. Bouyain S and Leahy DJ: Structure-based mutagenesis of the substrate-recognition domain of Nrdp1/FLRF identifies the binding site for the receptor tyrosine kinase ErbB3. Protein Sci 16: 654-661, 2007.

8. Meroni G and Diez-Roux G: TRIM/RBCC, a novel class of 'single protein RING finger' E3 ubiquitin ligases. Bioessays 27: 1147-1157, 2005.

9. Qiu XB and Goldberg AL: Nrdp1/FLRF is a ubiquitin ligase promoting ubiquitination and degradation of the epidermal growth factor receptor family member, ErbB3. Proc Natl Acad Sci USA 99: 14843-14848, 2002.

10. Yen L, Cao Z, Wu X, Ingalla ER, Baron C, Young LJ, Gregg JP, Cardiff RD, Borowsky AD, Sweeney C and Carraway KL III: Loss of Nrdp1 enhances ErbB2/ErbB3-dependent breast tumor cell growth. Cancer Res 66: 11279-11286, 2006.

11. Lu H, Li H, Mao D, Zhu Z and Sun H: Nrdp1 inhibits growth of colorectal cancer cells by nuclear retention of $\mathrm{p} 27$. Tumour Biol 35: 8639-8643, 2014.

12. Qiu XB, Markant SL, Yuan J and Goldberg AL: Nrdp1-mediated degradation of the gigantic IAP, BRUCE, is a novel pathway for triggering apoptosis. EMBO J 23: 800-810, 2004.

13. Yu F and Zhou J: Parkin is ubiquitinated by Nrdp1 and abrogates Nrdp1-induced oxidative stress. Neurosci Lett 440: 4-8, 2008.

14. Wang C, Chen T, Zhang J, Yang M, Li N, Xu X and Cao X: The E3 ubiquitin ligase Nrdp1 'preferentially' promotes TLR-mediated production of type I interferon. Nat Immunol 10: 744-752, 2009.

15. Chen Z, Naito M, Hori S, Mashima T, Yamori T and Tsuruo T: A human IAP-family gene, apollon, expressed in human brain cancer cells. Biochem Biophys Res Commun 264: 847-854, 1999.

16. Bartke T, Pohl C, Pyrowolakis G and Jentsch S: Dual role of BRUCE as an antiapoptotic IAP and a chimeric E2/E3 ubiquitin ligase. Mol Cell 14: 801-811, 2004.

17. Qiu XB and Goldberg AL: The membrane-associated inhibitor of apoptosis protein, BRUCE/Apollon, antagonizes both the precursor and mature forms of Smac and caspase-9. J Biol Chem 280: 174-182, 2005.

18. Hao Y, Sekine K, Kawabata A, Nakamura H, Ishioka T, Ohata H, Katayama R, Hashimoto C, Zhang X, Noda T, et al: Apollon ubiquitinates SMAC and caspase-9, and has an essential cytoprotection function. Nat Cell Biol 6: 849-860, 2004 
19. Shi H, Du J, Wang L, Zheng B, Gong H, Wu Y, Tang Y, Gao Y and Yu R: Lower expression of Nrdp1 in human glioma contributes tumor progression by reducing apoptosis. IUBMB Life 66: 704-710, 2014

20. Livak KJ and Schmittgen TD: Analysis of relative gene expression data using real-time quantitative PCR and the 2(-Delta Delta C(T)) Method. Methods 25: 402-408, 2001.

21. Valsechi MC, Oliveira AB, Conceição AL, Stuqui B Candido NM, Provazzi PJ, de Araújo LF, Silva WA Jr, Calmon Mde F and Rahal P: GPC3 reduces cell proliferation in renal carcinoma cell lines. BMC Cancer 14: 631, 2014.

22. Qiao HP, Gao WS, Huo JX and Yang ZS: Long non-coding RNA GAS5 functions as a tumor suppressor in renal cell carcinoma. Asian Pac J Cancer Prev 14: 1077-1082, 2013.

23. Zhang T, Zheng J, Jiang N, Wang G, Shi Q, Liu C and Lu Y: Overexpression of DLC-1 induces cell apoptosis and proliferation inhibition in the renal cell carcinoma. Cancer Lett 283: 59-67, 2009.
24. Hu G, Lai P, Liu M, Xu L, Guo Z, Liu H, Li W, Wang G, Yao X, Zheng J and Xu Y: MiR-203a regulates proliferation, migration, and apoptosis by targeting glycogen synthase kinase-3 $\beta$ in human renal cell carcinoma. Tumour Biol 35: 11443-11453, 2014.

25. Hassan M, Watari H, AbuAlmaaty A, Ohba Y and Sakuragi N: Apoptosis and molecular targeting therapy in cancer. Biomed Res Int 2014: 150845, 2014

26. Wong RS: Apoptosis in cancer: From pathogenesis to treatment. J Exp Clin Cancer Res 30: 87, 2011.

27. Bartke T, Pohl C, Pyrowolakis G and Jentsch S: Dual role of BRUCE as an antiapoptotic IAP and a chimeric E2/E3 ubiquitin ligase. Mol Cell 14: 801-811, 2004.

28. Ren J, Shi M, Liu R, Yang QH, Johnson T, Skarnes WC and Du C: The Birc6 (Bruce) gene regulates p53 and the mitochondrial pathway of apoptosis and is essential for mouse embryonic development. Proc Natl Acad Sci USA 102: 565-570, 2005. 